\title{
DISTANCES TO GALACTIC HIGH-VELOCITY CLOUDS: COMPLEX C
}

\author{
B. P. Wakker, ${ }^{1}$ D. G. York, ${ }^{2}$ J. C. Howk,${ }^{3}$ J. C. Barentine, ${ }^{4}$ R. Wilhelm, ${ }^{5}$ R. F. Peletier,${ }^{6}$ H. van Woerden, ${ }^{6}$ \\ T. C. BeErs, ${ }^{7}$ Ž. Ivezić, ${ }^{8}$ P. Richter, ${ }^{9}$ AND U. J. Schwarz ${ }^{6,10}$ \\ Received 2007 September 5; accepted 2007 October 8; published 2007 November 1
}

\begin{abstract}
We report the first determination of a distance bracket for the high-velocity cloud (HVC) complex C. Combined with previous measurements showing that this cloud has a metallicity of 0.15 times solar, these results provide ample evidence that complex $\mathrm{C}$ traces the continuing accretion of intergalactic gas falling onto the Milky Way. Accounting for both neutral and ionized hydrogen as well as He, the distance bracket implies a mass of (3-14) $\times 10^{6} M_{\odot}$, and the complex represents a mass inflow of $0.1-0.25 M_{\odot} \mathrm{yr}^{-1}$. We base our distance bracket on the detection of $\mathrm{Ca}$ II absorption in the spectrum of the blue horizontal branch (BHB) star SDSS J120404.78+623345.6, in combination with a significant nondetection toward the BHB star BS 16034-0114. These results set a strong distance bracket of 3.7-11.2 kpc on the distance to complex C. A more weakly supported lower limit of $6.7 \mathrm{kpc}$ may be derived from the spectrum of the BHB star BS 16079-0017.
\end{abstract}

Subject headings: Galaxy: evolution — Galaxy: general — Galaxy: halo — Galaxy: structure — ISM: clouds — stars: distances

\section{INTRODUCTION}

The evolution of galaxies is strongly driven by the gas in the interstellar medium. There is strong evidence for the infall of new material that provides fuel for galaxy growth. This gas may originate in accreted satellite galaxies, as gas tidally pulled out of passing galaxies, or from pristine intergalactic gas. The cool, infalling clouds appear to be embedded in an extended (100-200 kpc radius) hot corona (Sembach et al. 2003). Indirect evidence for infalling gas is provided by two arguments: (1) At the current rate of star formation, all of the ISM will be turned into stars within about a Gyr. (2) The narrowness of the distribution of metallicities of long-lived stars implies that the metallicity of the ISM remains more or less constant over a Hubble time, which can happen if there is a continuing inflow of low-metallicity material with a present-day rate of about 1 $M_{\odot} \mathrm{yr}^{-1}$. Item 2 is known as the "G-dwarf problem" (van den Bergh 1962). Using the infall hypothesis to solve it has been the subject of much theoretical work (see, e.g., Pagel 1997 for a good summary). Continuing infall is essential in detailed numerical modeling of the chemical evolution of the Galaxy and the development of abundance gradients (e.g., Chiappini et al. 2001 and references therein). Infall of low-metallicity gas

\footnotetext{
${ }^{1}$ Department of Astronomy, University of Wisconsin, Madison, WI 53706; wakker@astro.wisc.edu.

${ }^{2}$ Astronomy and Astrophysics Center, University of Chicago, Chicago, IL 60637; don@oddjob.uchicago.edu.

${ }^{3}$ Department of Physics, University of Notre Dame, Notre Dame, IN 46556; jhowk@nd.edu.

${ }^{4}$ Department of Astronomy, University of Texas, Austin, TX 78712; and Apache Point Observatory, Sunspot, NM 88349; jcb@astro.as.utexas.edu.

${ }^{5}$ Department of Physics and Astronomy, Texas Tech University, Lubbock, TX 79409; ron.wilhelm@ttu.edu.

${ }^{6}$ Kapteyn Astronomical Institute, University of Groningen, Postbus 800, 9700 AV, Groningen; peletier@astro.rug.nl, hugo@astro.rug.nl.

${ }^{7}$ Department of Physics and Astronomy, CSCE (Center for the Study of Cosmic Evolution), and JINA (Joint Institute for Nuclear Astrophysics), Michigan State University, East Lansing, MI 48824; beers@pa.msu.edu.

${ }^{8}$ Department of Astronomy, University of Washington, Box 351580, Seattle, WA 98195; ivezic@astro.washington.edu.

${ }^{9}$ Institut für Physik, Universität Potsdam, Am Neuen Palais 10, 14469 Potsdam, Germany; prichter@astro.physik.uni-potsdam.de.

${ }^{10}$ Department of Astrophysics, Radboud University Nijmegen, Toernooiveld 1, NL-6525 ED, Netherlands; schwarz@kun.nl.
}

also seems necessary to reproduce the relatively high abundance of deuterium measured in the local interstellar medium (Linksy et al. 2006).

Direct observational evidence for infalling low-metallicity gas is provided by the high-velocity clouds (HVCs; see reviews by Wakker \& van Woerden 1997; Richter 2006). Subsolar metallicities have now been determined for 11 clouds (see van Woerden \& Wakker 2004 for a summary). In particular, the metallicity of complex $\mathrm{C}$ is well established as 0.15 times solar (see summary by Fox et al. 2004). Complex C also has a high deuterium abundance (Sembach et al. 2004). Distance brackets have been more elusive, with just one known before 2006 (8$10 \mathrm{kpc}$ for complex A; van Woerden et al. 1999b; Wakker et al. 2003). Thom et al. (2006) derive an $8.8 \mathrm{kpc}$ upper limit for cloud WW 35, while in a separate paper (Wakker et al. 2007, hereafter Paper I), we present new results for two HVCs (9.8$15.1 \mathrm{kpc}$ for complex GCP and 5.0-11.7 kpc for the Cohen Stream). In this Letter we report a distance bracket for the HVC covering the largest sky area: complex $\mathrm{C}$. We summarize our method in $\S 2$. The data are described in $\S 3$ and the results in $\S 4$, while in $\S 5$ we summarize the implications.

\section{METHOD}

To find the distance to a HVC, we search for interstellar absorption at the cloud's velocity in spectra of stars with known distances. A detection sets an upper limit, while a significant nondetection sets a lower limit. A significant nondetection means that the ratio of the expected equivalent width to the observed $3 \sigma$ upper limit is sufficiently large (e.g., $>3$; see Appendix item 15 in Wakker 2001). We also refer to Paper I for a detailed discussion.

We find probe stars from the HK survey (Beers et al. 1996), the Sloan Digital Sky Survey (SDSS; Fukugita et al. 1996; Gunn et al. 1998, 2006; York et al. 2000; Stoughton et al. 2002; Pier et al. 2003; Adelman-McCarthy et al. 2007), and the 2MASS survey (Cutri et al. 2003; Brown et al. 2004). Using color criteria we identify blue horizontal branch (BHB) and RR Lyrae candidates, for which we then obtain intermediateresolution spectra and photometry to derive the stellar parameters. See Paper I for more details and R. Wilhelm et al. (2008, in preparation) for a complete description. 


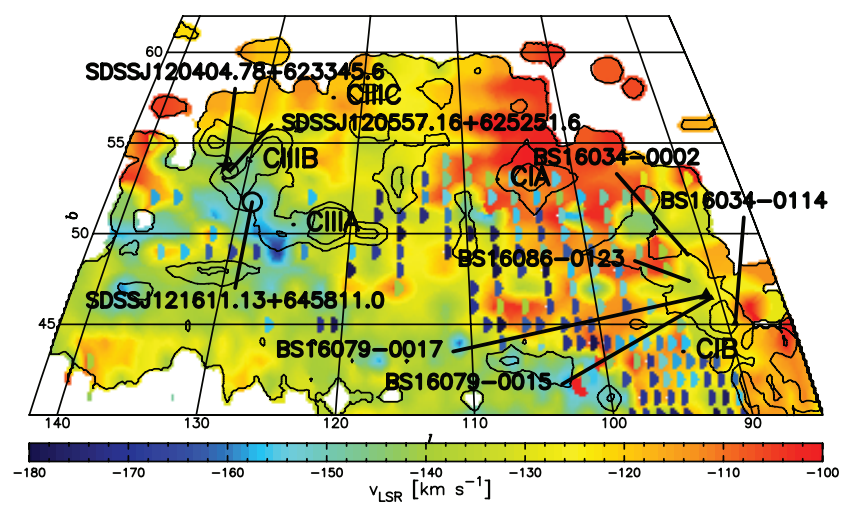

FIG. 1.-H I map of complex C, based on the data of Hulsbosch \& Wakker (1988). Colors represent LSR velocities, as coded in the wedge. Half-circles show positions with multiple $\mathrm{H}$ I components. Contour levels are at brightness temperatures of $0.05,0.25,0.5$, and $1 \mathrm{~K}$. The positions of the stars discussed in this Letter are shown by the symbols: filled stars for the detections, filled triangles for the significant nondetections, and open circles for nonsignificant nondetections. The symbol diameters are proportional to the stellar distances. Several cores inside complex $\mathrm{C}$ are labeled.

\section{OBSERVATIONS}

\subsection{WHT Observations}

In 1997, we selected several stars with estimated distances between 2 and $8 \mathrm{kpc}$ projected on the complex $\mathrm{C}$ core $\mathrm{C}$ I (Fig. 1). Five of these were observed by Peletier and van Woerden with the $4.2 \mathrm{~m}$ William Herschel Telescope (WHT) on La Palma on 1997 May 17/18/19, using the Utrecht Echelle Spectrograph (UES) at the WHT Nasmyth focus. The spectra covered the wavelength range from 3610 to $4510 \AA$, with a velocity resolution of $6 \mathrm{~km} \mathrm{~s}^{-1}$. The wavelength calibration was obtained with a ThAr lamp. The standard IRAF reduction included bias and flat-field corrections, summing of pixels across the spectrum, and sky subtraction.

A preliminary analysis of these spectra and preliminary distances to the stars yielded a lower limit of $6.1 \mathrm{kpc}$ to the distance of complex C (van Woerden et al. 1999a; Wakker 2001). We have now derived final stellar distances for three stars, using photometric data and intermediate-resolution spectra obtained at the McDonald Observatory $2.7 \mathrm{~m}$ telescope. Table 1 presents the basic data for these stars. All three are hot, high-gravity stars, and we carefully checked the spectral details against the derived stellar parameters. For one star (BS 16086-0123) we do not have classification data, and one star (BS 16034-0002) turns out to be a cool, nearby star.

\subsection{Keck Observations}

In 2007, we selected several stars from the SDSS that lie projected onto core C III (see Fig. 1), with distances ranging from 9.3 to $40.6 \mathrm{kpc}$. These distances are based on SDSS spectroscopic and photometric data. On 2007 April 24, Barentine observed three of these using the upgraded High Resolution Echelle Spectrometer (HIRES; Vogt et al. 1994) on the Keck I telescope. The 2004 HIRES upgrade replaced the single CCD with a three-CCD mosaic, including two with enhanced blue sensitivity. All data were collected using the UV cross disperser and the C5 decker (1.15" wide slit). The data were binned by 2 pixels $\left(0.24^{\prime \prime}\right)$ in the spatial direction. The seeing was approximately $1.5^{\prime \prime}$. The spectra cover the wavelength range 3380 to $4330 \AA$ and have a spectral resolution of $8.8 \mathrm{~km}$ $\mathrm{s}^{-1}$. Three stars could be observed before weather conditions forced the telescope to close for the night. Two are useful (see Table 1), while for SDSS J120557.16+625251.6 the stellar $\mathrm{Ca}$ II line obscures the interstellar lines.

The spectra were extracted by Howk using the HIRedux package (ver. 2.2) of J. X. Prochaska. ${ }^{11}$ The two-dimensional echelle images were bias-subtracted, flat-fielded, and wavelength-calibrated using the HIRES ThAr and quartz (flat field) lamps. One-dimensional spectra are extracted using an optimal extraction routine, and individual exposures and orders were co-added with an inverse variance weighting. The blaze function of the instrument was removed (before co-adding orders) by fitting a polynomial to the average flux distribution of the orders within each of the three CCDs.

\subsection{H I Data}

We also have $21 \mathrm{~cm} \mathrm{H}$ I profiles toward the probe stars. Effelsberg spectra $\left(9.7^{\prime}, 1 \mathrm{~km} \mathrm{~s}^{-1}\right.$ resolution; Wakker et al.

${ }^{11}$ Available at http://www.ucolick.org/ xavier/IDL/.

TABLE 1

STellar and Interstellar Data

\begin{tabular}{|c|c|c|c|c|c|c|c|c|c|c|c|c|}
\hline $\begin{array}{l}\text { Object } \\
\text { (1) }\end{array}$ & $\begin{array}{c}l \\
\text { (deg) } \\
(2)\end{array}$ & $\begin{array}{c}b \\
(\mathrm{deg}) \\
(3)\end{array}$ & $\begin{array}{l}\text { Distance } \\
(\mathrm{kpc}) \\
(4)\end{array}$ & $\begin{array}{c}v_{*} \\
\left(\mathrm{~km} \mathrm{~s}^{-1}\right) \\
(5)\end{array}$ & $\begin{array}{l}T_{\text {exp }} \\
(\mathrm{s}) \\
(6)\end{array}$ & $\begin{array}{l}\mathrm{S} / \mathrm{N} \\
(7)\end{array}$ & $\begin{array}{c}v_{\mathrm{HVC}} \\
\left(\mathrm{km} \mathrm{s}^{-1}\right) \\
(8)\end{array}$ & $\begin{array}{c}N(\mathrm{H} \mathrm{I}) \\
\left(10^{18} \mathrm{~cm}^{-2}\right) \\
(9)\end{array}$ & $\begin{array}{c}W_{\text {exp }}(\mathrm{K}) \\
(\mathrm{m} \AA) \\
(10)\end{array}$ & $\begin{array}{c}W_{\text {exp }}(\mathrm{H}) \\
(\mathrm{m} \AA) \\
(11)\end{array}$ & $\begin{array}{c}W_{\mathrm{obs}}(\mathrm{K}) \\
(\mathrm{m} \AA) \\
(12)\end{array}$ & $\begin{array}{c}W_{\text {obs }}(\mathrm{H}) \\
(\mathrm{m} \AA) \\
(13)\end{array}$ \\
\hline \multicolumn{13}{|l|}{ SDSS: } \\
\hline $\mathrm{J} 121611.13+645811.0$ & 128.94 & 51.74 & $12.5 \pm 0.4$ & -306 & 1000 & 8 & $\begin{array}{r}-156 \\
-51\end{array}$ & $\begin{array}{r}59.3 \pm 1.1 \\
127.2 \pm 2.5\end{array}$ & $\begin{array}{l}106 \\
215\end{array}$ & $\begin{array}{r}62 \\
161\end{array}$ & $\begin{array}{c}<43 \\
210+11+4\end{array}$ & $\begin{array}{c}<49 \\
122+16+5\end{array}$ \\
\hline $\mathrm{J} 120404.78+623345.6$ & 132.12 & 53.71 & $10.9 \pm 0.7$ & -389 & 1600 & 20 & $\begin{array}{r}-145 \\
-48\end{array}$ & $\begin{array}{r}31.2 \pm 0.8 \\
103.1 \pm 1.3\end{array}$ & $\begin{array}{r}42 \\
186\end{array}$ & $\begin{array}{r}23 \\
130\end{array}$ & $\begin{array}{c}42 \pm 3 \pm 4 \\
\ldots\end{array}$ & $\begin{array}{c}19 \pm 6 \pm 3 \\
113 \pm 15 \pm 3\end{array}$ \\
\hline \multicolumn{13}{|l|}{ BS: } \\
\hline $16079-0017$ & 91.05 & 46.60 & $8.1 \pm 2.9$ & -208 & 1800 & $20^{\mathrm{a}}$ & -139 & $32.5 \pm 1.2$ & 45 & 24 & $<16$ & \\
\hline $16034-0114^{\mathrm{b}}$ & 89.39 & 45.07 & $3.8 \pm 0.3$ & 40 & 3600 & 16 & -123 & $71.6 \pm 1.3$ & 132 & 82 & $<12$ & $<21$ \\
\hline $16079-0015^{\mathrm{c}}$ & 90.69 & 46.46 & $2.0 \pm 0.2$ & -234 & 1800 & $62^{\mathrm{d}}$ & -134 & $50.1 \pm 3.0$ & 85 & 48 & $<8$ & $\ldots$ \\
\hline
\end{tabular}

Notes. - Cols. (2) and (3): Galactic longitude and latitude of the stars. Col. (4): Distance as determined by R. Wilhelm et al. (2008, in preparation). Col. (5): Stellar velocity (relative to the LSR), as measured from Ca II and Fe I lines. Col. (6): Exposure time with the WHT (BS stars) or Keck (SDSS stars). Col. (7): S/N ratio in Ca II K near the HVC velocity. Col. (8) and (9): Velocity (relative to the LSR) and H I column density of complex C in the direction of the star. Values are based on the LAB survey (Kalberla et al. 2005) for SDSS stars, and on our Effelsberg data (Wakker et al. 2001) for BS stars. Cols. (10) and (11): Expected $\mathrm{K}$ and $\mathrm{H}$ equivalent widths, using the relation $\log [N(\mathrm{Ca} \mathrm{II}) / N(\mathrm{H} \mathrm{I})]=-7.76-0.78\{\log [N(\mathrm{H} \mathrm{I})]-19.5\}$ found by Wakker \& Mathis $(2000)$. Note that the uncertainty in $N(\mathrm{H} \mathrm{I})$ due to the large radio beam size produces an uncertainty of about $20 \mathrm{~m} \AA$ in these predictions. Cols. (12) and (13): Observed K and $\mathrm{H}$ equivalent widths or $3 \sigma$ upper limits.

${ }^{\text {a }}$ There are too many stellar lines to calculate a $\mathrm{Ca}$ II $\mathrm{H}$ error

${ }^{\mathrm{b}}$ Alternative names 2MASS J160007.91+575125.4 and BS 16079-0065.

${ }^{c}$ Alternative name 2MASS J154747.53+580646.4.

${ }^{\mathrm{d}}$ The error used for calculating the $\mathrm{S} / \mathrm{N}$ includes variations due to many weak stellar lines. 


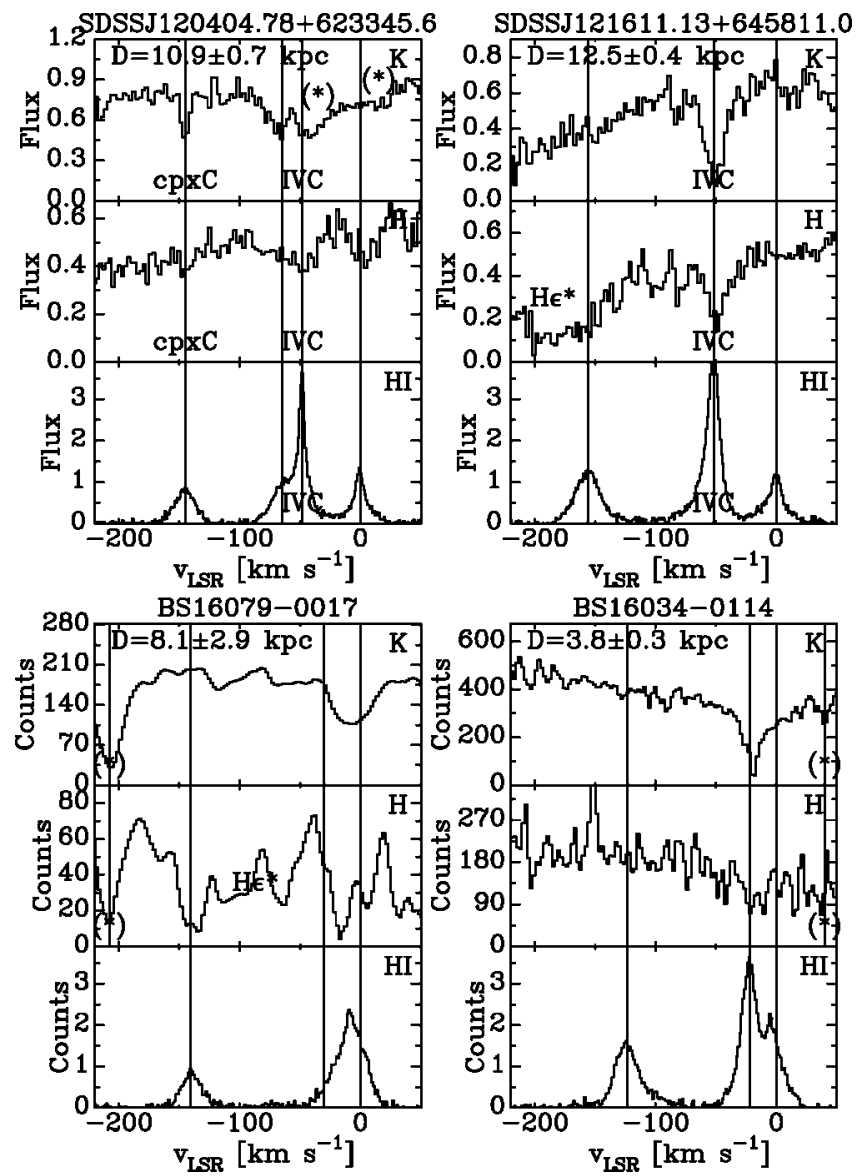

FIG. 2.- Spectra near Ca II K, Ca II H, and $\mathrm{H}$ I for the four most significant stars in our sample. Note that the Keck data are flux-calibrated (in units of $10^{-15} \mathrm{ergs} \mathrm{cm}^{-2} \mathrm{~s}^{-1} \AA^{-1}$ ), while the WHT data are not. Vertical lines are placed at the velocity of the low-, intermediate-, and high-velocity $\mathrm{H}$ I emission components, while detected absorption lines are labeled "cpxC" and "IVC". The locations of stellar Ca II and Fe II lines are shown by the asterisk (*). Note that near the $\mathrm{H}$ line toward BS 16079-0017 there are many unidentified stellar lines.

2001) are available for the stars observed with the WHT. For the Keck stars we use the LAB data set of Kalberla et al. (2005; $36^{\prime}, 1 \mathrm{~km} \mathrm{~s}^{-1}$ resolution). However, Wakker et al. (2001) found that $N(\mathrm{H} \mathrm{I})$ measured with a $36^{\prime}$ beam can be up to a factor 2.5 larger or smaller than $N\left(\mathrm{H}\right.$ I) measured with a $9.7^{\prime}$ beam; the ratio distribution has a mean of 1 and rms of 0.2 . The $\mathrm{H}$ I column densities therefore have a large $(>20 \%)$ systematic uncertainty. Higher resolution observations $\left(\sim 1^{\prime}\right)$ with a synthesis telescope are needed to obtain more accurate values.

\section{RESULTS}

Columns (8)-(13) of Table 1 list the $\mathrm{H}$ I and Ca II measurements, including predictions for the equivalent width (EW) based on the relation between $N(\mathrm{H} \mathrm{I})$ and $\mathrm{Ca}$ II abundance found by Wakker \& Mathis (2000; see notes to Table 1). Figure 2 shows the $\mathrm{Ca}$ II $\mathrm{K}$ and $\mathrm{H}$ and $\mathrm{H}$ I $21 \mathrm{~cm}$ spectra for the four stars that yield significant results.

We detect $\mathrm{Ca}$ II $\mathrm{K}$ and $\mathrm{H}$ absorption associated with complex C toward the star SDSS J120404.78+623345.6, with EW(K) $=42 \pm 3 \pm 4 \mathrm{m \AA}$ and $\mathrm{EW}(\mathrm{H})=19 \pm 6 \pm 3 \mathrm{m \AA}$. The first error is statistical error associated with the noise in the spectrum and the placement of the continuum. The second error is a systematic error associated with a $3 \mathrm{~km} \mathrm{~s}^{-1}$ uncertainty in choosing the velocity limits of the equivalent width integration. See Wakker et al. (2003) for a full discussion of these errors.

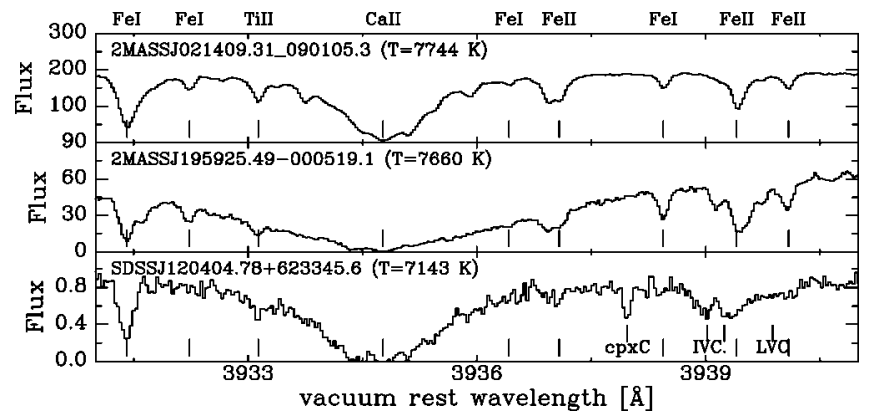

FIG. 3.- Spectra of three stars, shifted to the stellar reference frame. The top two (2MASS) stars were observed with the VLT in Paper I. Most of the stellar lines can be identified as Fe I or Fe II absorption. Some features have not yet been identified. Clearly, no stellar line is expected at the wavelength of the complex $\mathrm{C} \mathrm{Ca}$ II $\mathrm{K}$ absorption. The two-component intermediate-velocity and low-velocity $\mathrm{Ca}$ II $\mathrm{K}$ absorption in the direction of SDSS J120404.78+623345.6 blends with stellar Fe II absorption.

That this line is interstellar is shown by two facts. (1) It is much narrower $\left(\mathrm{FWHM}=6.7 \mathrm{~km} \mathrm{~s}^{-1}\right)$ than the stellar lines (FWHM $\sim 15 \mathrm{~km} \mathrm{~s}^{-1}$ ). (2) Stars with effective temperatures of about $7000 \mathrm{~K}$ do not have a stellar line at this location (see Fig. 3). The distance of SDSS J120404.78+623345.6 is found to be $10.9 \pm 0.7 \mathrm{kpc}$. As we discuss in Paper I, this implies a $68 \%$ confidence interval for the upper limit on the distance of complex C of $D_{*}+0.47 \sigma\left(D_{*}\right)=11.2 \mathrm{kpc}$.

The three WHT stars yield nondetections for Ca II in complex C. The spectrum of BS $16079-0017(D=8.1 \pm 2.9 \mathrm{kpc})$ shows many broad stellar lines, but no narrow interstellar line is visible at the velocity of complex $\mathrm{C}$. This star thus sets a tentative lower limit of $6.7 \mathrm{kpc}$ to the distance of complex C. On the other hand, a firm lower limit of $3.7 \mathrm{kpc}$ is set by BS 16034-0114 $(D=3.8 \pm 0.3 \mathrm{kpc})$, whose spectrum shows few stellar lines and which has EW(expected) $/ 3 \sigma(\mathrm{EW})=11$. The star BS 16079-0015 also yields a significant non-detection, but since it is closer than BS 16034-0114, we do not show its spectrum in Figure 2.

Complex $\mathrm{C}$ is also not detected toward the star SDSS $\mathrm{J} 121611.13+645811.0$, even though this star is more distant than SDSS J120404.78+623345.6. However, the expected EW is only a factor 2.5 higher than the $3 \sigma$ limit. Considering possible intrinsic variations in the $\mathrm{Ca}$ II abundance, and the large uncertainty in the $\mathrm{H}$ I column density (see above), this nondetection is not considered significant, although only marginally so. In fact, there is a hint of an interstellar absorption line at the velocity of complex C (see Fig. 2). Data with higher $\mathrm{S} / \mathrm{N}$ ratio are needed to resolve this issue.

\section{CONCLUSIONS}

Forty years after the first attempt (Prata \& Wallerstein 1967), we report the first successful detection of interstellar $\mathrm{Ca}$ II $\mathrm{H}$ and $\mathrm{K}$ absorption from HVC complex C. This sets an upper limit on the distance of core C III (left side of Fig. 1) of $11.2 \mathrm{kpc}$. For core C I (right side of Fig. 1) we find a lower limit of $3.7 \mathrm{kpc}$, possibly $6.7 \mathrm{kpc}$. Although the stars are $27^{\circ}$ apart on the sky, it is still safe to conclude that complex $\mathrm{C}$ is located at Galactocentric radius $<14 \mathrm{kpc}$, and lies high above the Galactic plane $(z=3-9 \mathrm{kpc})$. A more precise determination requires a lower limit for core $\mathrm{C}$ III and an upper limit for C I.

Integrating $N(\mathrm{H} \mathrm{I})$ across the cloud, we estimate $M(\mathrm{H} \mathrm{I})$ as (0.7-6) $\times 10^{6} M_{\odot}$. H $\alpha$ emission has also been detected (Tufte et al. 1998). We can assume either that the $\mathrm{H}^{+}$and $\mathrm{H} \mathrm{I}$ are thoroughly mixed or that the $\mathrm{H}^{+}$originates in a photoionized 
skin around the cloud. In either case, the observed $\mathrm{H} \alpha$ intensity suggests that there is roughly as much ionized as neutral gas.

We can also estimate the mass inflow associated with complex $\mathrm{C}$, using a method described in Paper I. Including the neutral and ionized hydrogen, as well as a $40 \%$ contribution from helium, we derive that complex $\mathrm{C}$ represents about $0.1-$ $0.25 M_{\odot} \mathrm{yr}^{-1}$ of infalling gas. This is a substantial fraction of the theoretically required amount of $1 M_{\odot} \mathrm{yr}^{-1}$. Other HVCs may contribute the rest, but we have not yet determined distances and metallicities for the most likely candidates.

From our results, we conclude that the mystery of the distances to the HVCs is beginning to be solved. The evidence shows that several HVCs are located in the upper reaches of the gaseous Galactic halo and that they contribute significantly to the inflow of metal-poor gas onto the Galaxy. Once the mass inflow rate is constrained from observations of a sufficient number of HVCs, the next step will be to determine their threedimensional structure, so that we can use their velocities and Galactic location to derive orbits and solve the outstanding mystery of their ultimate origins.

B. P. W., D. G. Y., R. W., and T. C. B. acknowledge support from grant AST 06-07154 awarded by the US National Science Foundation. T. C. B. also acknowledges NSF grants AST 0406784 and PHY 02-16783, and the Physics Frontier Center/ Joint Institute for Nuclear Astrophysics (JINA).

Some of the data presented were obtained at the W. M. Keck Observatory, which is operated as a scientific partnership among the California Institute of Technology, the University of California, and the National Aeronautics and Space Ad- ministration. The Observatory was made possible by the generous financial support of the W. M. Keck Foundation.

The William Herschel telescope is operated on the Island of La Palma by the Isaac Newton group in the Spanish Observatorio del Roque de los Muchachos of the Instituto de Astrophysica de Canarias.

Funding for the SDSS and SDSS-II has been provided by the Alfred P. Sloan Foundation, the Participating Institutions, the National Science Foundation, the US Department of Energy, the National Aeronautics and Space Administration, the Japanese Monbukagakusho, the Max Planck Society, and the Higher Education Funding Council for England. The SDSS Web site is http://www.sdss.org/. The SDSS is managed by the Astrophysical Research Consortium for the Participating Institutions. The Participating Institutions are the American $\mathrm{Mu}-$ seum of Natural History, Astrophysical Institute Potsdam, University of Basel, University of Cambridge, Case Western Reserve University, University of Chicago, Drexel University, Fermilab, the Institute for Advanced Study, the Japan Participation Group, the Johns Hopkins University, the Joint Institute for Nuclear Astrophysics, the Kavli Institute for Particle Astrophysics and Cosmology, the Korean Scientist Group, the Chinese Academy of Sciences (LAMOST), Los Alamos National Laboratory, the Max Planck Institute for Astronomy (MPIA), the Max Planck Institute for Astrophysics (MPA), New Mexico State University, Ohio State University, University of Pittsburgh, University of Portsmouth, Princeton University, the United States Naval Observatory, and the University of Washington.

\section{REFERENCES}

Adelman-McCarthy, J., et al. 2007, ApJS, 172, 634

Beers, T. C., Wilhelm, R., Doinidis, S. P., \& Mattson, C. J. 1996, ApJS, 103, 433

Brown, W. R., Geller, M. J., Kenyon, S. J., Beers, T. C., Kurtz, M. J., \& Roll, J. B. 2004, AJ, 127, 1555

Chiappini, C., Matteucci, F., \& Romano, D. 2001, ApJ, 554, 1044

Cutri, R. M., et al. 2003, 2MASS All Sky Catalog of Point Sources (Pasadena: IPAC)

Fox, A. J., Savage, B. D., Wakker, B. P., Richter, P., Sembach, K. R., \& Tripp, T. M. 2004, ApJ, 602, 738

Fukugita, M., Ichikawa, T., Gunn, J. E., Doi, M., Shimasaku, K., \& Schneider, D. P. 1996, AJ, 111, 1748

Gunn, J. E., et al. 1998, AJ, 116, 3040 2006, AJ, 131, 2332

Hulsbosch, A. N. M., \& Wakker, B. P. 1988, A\&A, 75, 191

Kalberla, P. W. W., Burton, W. B., Hartmann, D., Arnal, E. M., Bajaja, E., Morras, R., \& Pöppel, W. G. L. 2005, A\&A, 440, 775

Linsky, J. L., et al. 2006, ApJ, 647, 1106

Pagel, B. E. J. 1997, Nucleosynthesis and Chemical Evolution of Galaxies (Cambridge: Cambridge Univ. Press)

Pier, J. R., Munn, J. A., Hindsley, R. B., Hennessy, G. S., Kent, S. M., Lupton, R. H., \& Ivezić, Z. 2003, AJ, 125, 1559

Prata, S., \& Wallerstein, G. 1967, PASP, 79, 202

Richter, P. 2006, Rev. Mod. Astron., 19, 31

Sembach, K. R., et al. 2003, ApJS, 146, 165
Sembach, K. R., et al. 2004, ApJS, 150, 387

Stoughton, C., et al. 2002, AJ, 123, 485

Thom, C., Putman, M. E., Gibson, B. K., Christlieb, N., Flynn, C., Beers, T. C., Wilhelm, R., \& Lee, Y. S. 2006, ApJ, 638, L97

Tufte, S. L., Reynolds, R. J., \& Haffner, L. M. 1998, ApJ, 504, 773

van den Bergh, S. 1962, AJ, 67, 486

van Woerden, H., Peletier, R. F., Schwarz, U. J., Wakker, B. P., \& Kalberla, P. M. W. 1999a, in ASP Conf. Ser. 166, Stromlo Workshop on High-Velocity Clouds, ed. B. K. Gibson \& M. E. Putman (San Francisco: ASP), 1

van Woerden, H., Schwarz, U. J., Peletier, R. F., Wakker, B. P., \& Kalberla, P. M. W. 1999b, Nature 400, 138

van Woerden, H., \& Wakker, B. P. 2004, in High Velocity Clouds, ed. H. van Woerden, B. P. Wakker, U. J. Schwarz, \& K. S. de Boer (Dordrecht: Kluwer), 195

Vogt, S. S., et al. 1994, Proc. SPIE, 2198, 362

Wakker, B. P. 2001, ApJS, 136, 463

Wakker, B. P., Kalberla, P. M. W., van Woerden, H., de Boer, K. S., \& Putman, M. E. 2001, ApJS, 136, 537

Wakker, B. P., \& Mathis, J. S. 2000, ApJ, 544, L107

Wakker, B. P., \& van Woerden, H. 1997, ARA\&A, 35, 217

Wakker, B. P., York, D. G., Wilhelm, R., Barentine, J. C., Richter, P., Beers, T. C., Ivezić, Z., \& Howk, C. 2007, ApJ, submitted (Paper I)

Wakker, B. P., et al. 2003, ApJS, 146, 1

York, D. G., et al. 2000, AJ, 120, 1579 